\title{
Self-similar solutions of fully nonlinear curvature flows
}

\author{
JAMES AleXANDER MCCOY
}

\begin{abstract}
We consider closed hypersurfaces which shrink self-similarly under a natural class of fully nonlinear curvature flows. For those flows in our class with speeds homogeneous of degree 1 and either convex or concave, we show that the only such hypersurfaces are shrinking spheres. In the setting of convex hypersurfaces, we show under a weaker second derivative condition on the speed that again only shrinking spheres are possible. For surfaces this result is extended in some cases by a different method to speeds of homogeneity greater than 1 . Finally we show that self-similar hypersurfaces with sufficiently pinched principal curvatures, depending on the flow speed, are again necessarily spheres.
\end{abstract}

Mathematics Subject Classification (2010): 53C44 (primary), 35J60 (secondary).

\section{Introduction}

As part of his investigation into the asymptotic behaviour of the mean curvature flow of hypersurfaces with positive mean curvature, Huisken considered in [21] self-similar solutions which evolve by simple scaling. Such solutions satisfy the elliptic equation

$$
H=\langle X, v\rangle
$$

where $X, v$ and $H$ denote respectively the position vector, unit normal and mean curvature of the hypersurface and the inner product is the ordinary Euclidean dot product. Abresch and Langer [1] showed for dimension $n=1$ there is a two parameter family of closed immersed curves in $\mathbb{R}^{2}$ of positive geodesic curvature which are self similar solutions to the corresponding curvature flow in the plane.

The author is grateful for the support of an Australian Postdoctoral Fellowship from the Australian Research Council as part of a Discovery Grant entitled Singularities and surgery in curvature flows and for a University of Wollongong study leave assistance grant. The author also appreciates the support of the School of Mathematics and Applied Statistics at the University of Wollongong and the hospitality of the Centre for Mathematics and its Applications at the Australian National University, where part of this work was completed.

Received June 19, 2009; accepted in revised form March 5, 2010. 
Huisken showed in higher dimensions the sphere is the only compact hypersurface of positive mean curvature which moves under self similarities. Specifically:

Theorem 1.1 ([21]). Let $M$ be a compact $n$-dimensional hypersurface, $n \geq 2$, with nonnegative mean curvature $H$. If $M$ satisfies equation (1.1), then $M$ is a sphere of radius $\sqrt{n}$.

In this paper we establish the analogous result for a large class of fully nonlinear curvature flows. Although properties of fully nonlinear curvature flows have been considered before, as have self-similar solutions for various flows of curves ( $n=1$ ), for dimensions $n \geq 2$ very few results concerning compact self-similar solutions are known. In the case of mean curvature flow we have the result above for mean convex solutions, Angenent's "shrinking doughnuts" [12] and some results for arbitrary codimension [26]. Smoczyk also considered flows by functions of the mean curvature with some results on self-similar solutions [25]. Other results are self-similar convex solutions to flows by powers of the Gauss curvature ([5,7], see also [14] for classification of affine hyperspheres as ellipsoids) and convex solutions to flows of surfaces $(n=2)$ by powers $\alpha \in(0,1)$ of the harmonic mean curvature [3]. In this last case it was shown that such solutions are not always unique, so there are some nonspherical convex self-similar solutions in addition to spheres. In this paper we exploit maximum principle techniques in several settings to provide results for broad classes of fully nonlinear curvature flows rather than just specific flows. As in the case of the mean curvature flow, knowledge of self-similar solutions will be critical for future understanding of singularities in fully nonlinear curvature flows, a topic about which presently virtually nothing is known.

Our setting is a compact, $n$-dimensional manifold $M$, without boundary and a smooth immersion $X_{0}: M \rightarrow \mathbb{R}^{n+1}$ of $M$ as a hypersurface in Euclidean space. We take this $X_{0}$ as initial data and consider solutions to the curvature flow

$$
\frac{\partial X}{\partial t}(x, t)=-F^{\alpha}(\mathcal{W}(x, t)) v(x, t)
$$

where $\alpha \geq 1$ is a constant and $\mathcal{W}(x, t)$ denotes the matrix of the Weingarten map of the evolving hypersurface $M_{t}=X(\cdot, t)$ at the point $X(x, t)$.

The eigenvalues of the Weingarten map, $\kappa_{i}, i=1, \ldots, n$, are the principal curvatures of $M_{t}$ at the point $X(x, t)$. The mean curvature $H$ is the trace of the Weingarten map; the Gauss curvature is the determinant of $\mathcal{W}$.

The normal speed functions $F$ that we consider have the following properties:

\section{Condition 1.2.}

(i) $F(\mathcal{W})=f(\kappa(\mathcal{W}))$, where $f$ is a smooth, symmetric function of the eigenvalues $\kappa$ of $\mathcal{W}$.

(ii) $f$ is a smooth function defined on an open convex cone $\Gamma$ containing the positive cone $\Gamma^{+}=\left\{\kappa=\left(\kappa_{1}, \ldots, \kappa_{n}\right): \kappa_{i}>0\right.$ for all $\left.i\right\}$.

(iii) $f$ is strictly increasing in each argument: $\frac{\partial f}{\partial \kappa_{i}}>0$ everywhere in $\Gamma$. 
(iv) $f$ is positive and normalised such that $f(1, \ldots, 1)=1$.

(v) $f$ is homogeneous of degree 1: $f(k \kappa)=k f(\kappa)$ for any $k>0$ and all $\kappa \in \Gamma$.

Similar open cones $\Gamma$ larger than the positive cone have been considered in the context of expansion flows by Gerhardt [17] and by Urbas [29].

Condition 1.2, (iii) ensures (1.2) is parabolic. Short time existence of a solution to equation (1.2), where the initial data $M_{0}$ has principal curvatures lying inside the cone $\Gamma$ of definition of $F$, is well known (see, for example, [18]).

In the situation where the initial hypersurface $M_{0}$ is convex, much more is known about curvature contraction flows. For general positive homogeneity of the speed, Han showed the solution to the flow equation exists for a finite time and the hypersurface contracts to a point [19], generalising a similar result for the Gauss curvature flow by Chou [27]. For flows by powers of the mean curvature, Schulze showed additionally if the initial hypersurface satisfies a natural curvature condition, then the point is asymptotically round [24]. A similar result for flows by powers $\beta \geq \frac{1}{n}$ of the Gauss curvature was shown by Chow [15]. These generalise the famous result for the mean curvature flow by Huisken [20], although in that case and for some other flows no curvature pinching condition on the initial hypersurface is required. For other flows with speeds homogeneous of degree 1 there are many other results by Chow [16], Andrews [4, 5, 7, 8] and others. Recently, together with Andrews and $\mathrm{Yu}[11]$ the author further extended the known class of flow speeds for which convex initial data shrinks to an asymptotically round point. Andrews and the author have also recently broadened the class of flow speeds of homogeneity greater than 1 under which convex initial data satisfying a certain pinching condition shrinks to an asymptotically round point [10], generalising earlier results. Even more is known for surfaces, that is, when $n=2$, see e.g. [3, 6, 9, 23] and the appendix of [24]. We refer the reader to [24] for further discussion and references.

If we take an initial hypersurface $M_{0}=X_{0}(M)$ satisfying

$$
F^{\alpha}\left(\mathcal{W}_{0}\right)=\left\langle X_{0}, v_{0}\right\rangle
$$

where $F$ is homogeneous of degree $\alpha$, then it is easy to check that the homothetic deformation given by

$$
X(p, t)=\sqrt[\alpha+1]{(\alpha+1)(T-t)} X(p, 0)
$$

satisfies (1.2) up to tangential diffeomorphisms. The corresponding self-similar hypersurfaces $M_{t}$ shrink to a point at time $T$. Our self-similarity condition therefore corresponds to the elliptic equation

$$
F^{\alpha}(\mathcal{W})=\langle X, v\rangle
$$

the natural generalisation of (1.1). 
We prove the following analogues of Theorem 1.1:

Theorem 1.3. Let $M$ be a compact $n$-dimensional hypersurface, $n \geq 2$, without boundary. Suppose $M$ satisfies the self-similarity condition (1.3), where $F$ satisfies Conditions 1.2 and $\alpha=1$. If $f$ is concave, or, if $f$ is convex and $M$ has nonnegative mean curvature, then $M$ is a unit sphere.

\section{Remark 1.4.}

1. That $M$ satisfies (1.3) implies the curvatures of $M$ must lie within the cone $\Gamma$ of definition of $f$. This is analogous to the requirement of positive mean curvature in Theorem 1.1. Conceivably, it would suffice to have $f$ defined on a smaller open cone than $\Gamma^{+}$in which the curvatures of convex hypersurfaces $M$ under consideration lie, as in Theorem 1.7. One of our main interests in Theorem 1.3 however is that it allows hypersurfaces which are not necessarily convex.

2. Further to the previous remark, the proof of Theorem 1.3 in fact reveals a nonnegativity assumption on $F$ is sufficient, that is, a weakening of Conditions 1.2. However, the examples of $F$ we have in mind do not require this.

Examples of $F$ fitting the conditions of Theorem 1.3 include, once appropriately normalised:

- $F=E_{k}^{1 / k}$ for any $k=1, \ldots n$, where $E_{k}$ is the $k$ th elementary symmetric function of the principal curvatures of $M_{t}$,

$$
E_{k}=\sum_{1 \leq i_{1}<\ldots<i_{k} \leq n} \kappa_{i_{1}} \kappa_{i_{2}} \cdots \kappa_{i_{k}}
$$

In particular, $E_{1}=H=\operatorname{trace}(\mathcal{W}), E_{2}=R$ (the scalar curvature) and $E_{n}=$ $K=\operatorname{det}(\mathcal{W})$ (the Gauss curvature).

- The power means $F=H_{r}=\left(\frac{1}{n} \sum_{i=1}^{n} \kappa_{i}^{r}\right)^{\frac{1}{r}}$ for any $r$. The case of $r \rightarrow 0$ corresponds to $G=K^{\frac{1}{n}}$, as explained in [8].

- $F=\frac{E_{k}}{E_{k-1}}$, for any $k=2, \ldots, n$. $(k=1$ covered above $)$.

- $F=\left(\frac{E_{k}}{E_{l}}\right)^{\frac{1}{k-l}}, n \geq k>l \geq 0$.

- Positive linear combinations of convex examples or of concave examples from above.

The cone $\Gamma=\Gamma_{k}$ associated with examples above involving the elementary symmetric function $E_{k}$ is given by

$$
\left\{\kappa \in \mathbb{R}^{n}: E_{j}(\kappa)>0 \text { for all } j \leq k\right\} .
$$

For the less obvious verifications that these functions satisfy the conditions of the theorem, we refer the reader to $[8,22]$.

In the setting of strictly convex hypersurfaces and $\alpha=1$, we can obtain a similar result with more general positive linear combinations from the above list of examples using the Gauss map parametrisation. 
Theorem 1.5. If the hypersurface $M$ is strictly convex and satisfies (1.3) with $\alpha=$ 1 , where $F$ satisfies Conditions 1.2 and is inverse concave, then $M$ is a unit sphere.

The key in this case is inverse concavity of the speed $F$. In [8], $f$ was said to be inverse concave if the function

$$
\varphi\left(x_{1}, \ldots, x_{n}\right)=-f\left(\frac{1}{x_{1}}, \ldots, \frac{1}{x_{n}}\right)
$$

is concave. It was shown in that paper that inverse concavity of $f$ is equivalent to the matrix inequality:

$$
\frac{\partial^{2} f}{\partial x_{i} \partial x_{j}}+\frac{2}{x_{i}} \frac{\partial f}{\partial x_{i}} \delta_{i j} \geq 0 .
$$

In this paper, as in [11], we instead say that $f$ is inverse concave if the function

$$
\varphi\left(x_{1}, \ldots, x_{n}\right)=\frac{1}{f\left(\frac{1}{x_{1}}, \ldots, \frac{1}{x_{n}}\right)}
$$

is concave. It is straightforward to check that this definition is also equivalent to (1.4). However, advantages of this definition are that the function $\varphi$ is positive and homogeneous of degree 1 .

In this setting we work with convex hypersurfaces whose the principal radii of curvature are $r_{i}=\frac{1}{\kappa_{i}}, i=1, \ldots, n$. Then $\varphi\left(r_{1}, \ldots r_{n}\right)$ has a corresponding $\Phi\left(\mathcal{W}^{-1}\right)$ and its properties carry over from Conditions 1.2.

If $F$ is convex, then $F$ is necessarily inverse concave. The examples $E_{k}^{\frac{1}{k}}$ above are concave and inverse concave. Various others examples are also inverse concave, we refer the reader to [8] for details. Importantly, in the case of convex hypersurfaces, Theorem 1.5 allows positive linear combinations of inverse concave speeds, regardless of their individual convexity or concavity.

In the case of convex surfaces, $n=2$, we obtain additionally a result for speeds of higher homogeneity $\alpha \geq 1$, with no second derivative condition on $F$ required. For $\alpha>1$ we require the principal curvatures of the surface to satisfy a pinching condition, which, interestingly, is dependent only on $\alpha$.

Theorem 1.6. If the surface $M$ is closed, convex and satisfies the self-similarity condition (1.3), where $F$ satisfies Conditions 1.2 and $\alpha=1$, then $M$ is a unit sphere. If instead $\alpha>1$, then the result also holds provided the pinching ratio of the principal curvatures of $M$ satisfies

$$
\frac{\kappa_{\max }}{\kappa_{\min }} \leq 1+\frac{2}{\alpha-1} .
$$

Finally, we obtain a result in a similar spirit to Theorem 1.6 for the higher dimensional case, but for this we require the hypersurface to satisfy a pinching condition dependent on the particular speed $F$. However we require no second derivative condition on $F$ other a natural boundedness condition. 
Theorem 1.7. Let $M$ be a closed, convex $n$-dimensional hypersurface, $n \geq 2$. Suppose $M$ satisfies the self-similarity condition (1.3) where F satisfies Conditions 1.2 and $\alpha \geq 1$. If $M$ has sufficiently pinched principal curvatures, in the sense that there exists $\varepsilon \in[0,1]$, depending on $F$, such that

$$
\kappa_{i} \geq \varepsilon \kappa_{j},
$$

for all $i, j=1, \ldots, n$ then $M$ is a unit sphere.

Remark 1.8. Similar to Huisken's Theorem 1.1, Theorem 1.3 does not require the hypersurface $M$ to be convex, but does require $\alpha=1$ and $F$ either convex or concave. Of course $\alpha=1$ and these requirements on the speed are satisfied by the mean curvature $H$. Theorem 1.5 does require the hypersurface $M$ to be convex and $\alpha=1$, but needs only a weaker second derivative condition on $F$ and therefore broadens the class of applicable speeds. Theorem 1.6 only applies for convex surfaces $M$, but requires no second derivative condition on $F$, giving a result for $\alpha \geq 1$, albeit with an additional curvature pinching assumption on $M$ if $\alpha>1$. Finally, Theorem 1.7 applies for all convex hypersurfaces satisfying suitable curvature pinching dependent on the particular speed $F$.

We will use similar notation as in $[8,21,28]$. In particular, $g=\left\{g_{i j}\right\}, A=$ $\left\{h_{i j}\right\}$ and $\mathcal{W}=\left\{h_{j}^{i}\right\}$ denote respectively the metric, second fundamental form and Weingarten map of $M$. The mean curvature of $M$ is

$$
H=g^{i j} h_{i j}=h_{i}^{i}
$$

and the norm of the second fundamental form is

$$
|A|^{2}=g^{i j} g^{l m} h_{i l} h_{j m}=h_{l}^{j} h_{j}^{l}
$$

where $g^{i j}$ is the $(i, j)$-entry of the inverse of the matrix $\left(g_{i j}\right)$. In the last section we will also make use of the norm of the trace-free component of the second fundamental form,

$$
\left|A^{0}\right|^{2}=|A|^{2}-\frac{1}{n} H^{2}=\frac{1}{n} \sum_{i<j}\left(\kappa_{i}-\kappa_{j}\right)^{2},
$$

which is identically equal to zero when $M$ is a sphere, and the quantity

$$
C=\kappa_{1}^{3}+\ldots+\kappa_{n}^{3},
$$

where the letter $C$ is chosen simply by convention. Throughout this paper we sum over repeated indices from 1 to $n$ unless otherwise indicated. Raised indices indicate contraction with the metric. 
We will denote by $\left(\dot{F}^{k l}\right)$ the matrix of first partial derivatives of $F$ with respect to the components of its argument:

$$
\left.\frac{\partial}{\partial s} F(A+s B)\right|_{s=0}=\dot{F}^{k l}(A) B_{k l} .
$$

Similarly for the second partial derivatives of $F$ we write

$$
\left.\frac{\partial^{2}}{\partial s^{2}} F(A+s B)\right|_{s=0}=\ddot{F}^{k l, r s}(A) B_{k l} B_{r s} .
$$

Throughout the paper unless the argument is explicitly indicated we will always evaluate partial derivatives of $F$ at $\mathcal{W}$ and partial derivatives of $f$ at $\kappa(\mathcal{W})$. We will further use the shortened notation $\dot{f}^{i}=\frac{\partial f}{\partial \kappa_{i}}$ and $\dddot{f}^{i j}=\frac{\partial^{2} f}{\partial \kappa_{i} \partial \kappa_{j}}$ where appropriate.

The structure of this paper is straightforward: In Section 2 we prove Theorem 1.3, in Section 3 we prove Theorem 1.5, in Section 4 we prove Theorem 1.6 and in Section 5 we prove Theorem 1.7. The author would like to thank Ben Andrews for encouragement and suggestions which have helped him to strengthen the result in Section 3. The author is also grateful to Graham Williams, Glen Wheeler and Oliver Schnürer for useful discussions.

ACKNOWLEDGEMENTS. The author would like to thank the anonymous referee for comments which have led to improvements in the article.

\section{Proof of Theorem 1.3}

The argument of this section resembles that of Huisken in [21, Section 4]. Huisken used the maximum principle with the test function $\frac{|A|^{2}}{H^{2}}$; we use different test functions.

If, in addition to the requirements of the theorem, $F$ is strictly convex or strictly concave in nonradial directions, it suffices to use the test function $\frac{H}{F}$ and we consider this case first.

Differentiating covariantly (1.3) in an orthonormal frame $\left\{e_{i}\right\}$ on $M$, similarly as in [21], we have

$$
\nabla_{j} F=\dot{F}^{k l} \nabla_{j} h_{k l}=\left\langle X, h_{j}^{k} e_{k}\right\rangle
$$

and

$$
\nabla_{i} \nabla_{j} F=\ddot{F}^{k l, p q} \nabla_{i} h_{p q} \nabla_{j} h_{k l}+\dot{F}^{k l} \nabla_{i} \nabla_{j} h_{k l}=h_{i j}-F h_{i}{ }^{k} h_{j k}+\left\langle X, e_{k}\right\rangle \nabla^{k} h_{i j}
$$

where we have again substituted (1.3). 
Contracting (2.1) with $\dot{F}^{i j}$ yields

$$
\mathcal{L} F=\left(1-\dot{F}^{k l} h_{k m} h_{l}^{m}\right) F+\left\langle X, e_{k}\right\rangle \nabla^{k} F,
$$

where $\mathcal{L}$ denotes the elliptic operator $\dot{F}^{i j} \nabla_{i} \nabla_{j}$. That $F>0$ by Conditions 1.2, (iv) implies $H>0$ since $0<F \leq \frac{1}{n} H$ for $F$ concave. For a proof of this result we refer the reader to [28].

Alternatively, contracting (2.1) with the metric gives

$$
\ddot{F}^{k l, p q} \nabla^{i} h_{p q} \nabla_{i} h_{k l}+\dot{F}^{k l} \Delta h_{k l}=H-F|A|^{2}+\left\langle X, e_{k}\right\rangle \nabla^{k} H .
$$

Employing now Simons' identity

$$
\Delta h_{k l}=\nabla_{k} \nabla_{l} H-|A|^{2} h_{k l}+H h_{k}^{p} h_{p l},
$$

we obtain from (2.3) the equation

$$
\mathcal{L} H=\left(1-\dot{F}^{k l} h_{k m} h_{l}^{m}\right) H-\ddot{F}^{k l, p q} \nabla^{i} h_{p q} \nabla_{i} h_{k l}+\left\langle X, e_{k}\right\rangle \nabla^{k} H .
$$

In the case of $f$ convex and our assumption $H \geq 0$, the maximum principle applied to this equation reveals that in fact $H>0$. Thus $F>0$ since

$$
F \geq \frac{1}{n} H
$$

in this case.

It is now easy to compute using (2.2) and (2.4) that

$$
\mathcal{L}\left(\frac{H}{F}\right)=-\frac{1}{F} \ddot{F}^{k l, p q} \nabla^{i} h_{p q} \nabla_{i} h_{k l}+\left\langle X, e_{k}\right\rangle \nabla^{k}\left(\frac{H}{F}\right)-\frac{2}{F} \dot{F}^{k l} \nabla_{k} F \nabla_{l}\left(\frac{H}{F}\right) .
$$

In the case that the second order condition on $f$ is strict in nonradial directions, we have from [4, Lemma 7.12] that there exists a $C>0$ such that

$$
\ddot{F}^{k l, p q} \nabla^{i} h_{p q} \nabla_{i} h_{k l} \begin{cases}\geq C \frac{|\nabla A|^{2}}{|A|} & \text { if } f \text { is convex, } \\ \leq-C \frac{|\nabla A|^{2}}{|A|} & \text { if } f \text { is concave. }\end{cases}
$$

We remark that the above was derived in [4] for convex hypersurfaces, however, the inequalities hold more generally away from points with curvatures of the form $\left(\kappa_{1}, 0, \ldots, 0\right)$. Away from any potential such points, substituting either case of (2.6) into (2.5) we obtain by the strict elliptic maximum principle that $\frac{H}{F}$ is identically constant on a subset of $M$ which is both open and closed, hence $\frac{H}{F}$ is identically 
constant on $M$. Furthermore, (2.5) and (2.6) then imply $|\nabla A| \equiv 0$, so $M$ is a sphere. In view of the normalisation of $f$, the sphere has radius 1 .

In the more general setting where the convexity or concavity of $f$ is not necessarily strict in nonradial directions, we proceed as follows. Again using (2.1) and Simons' identity, a general homogeneous of degree zero symmetric function $G(\mathcal{W})=g(\kappa)$ satisfies

$$
\mathcal{L} G=\left(\dot{F}^{i j} \ddot{G}^{k l, r s}-\dot{G}^{i j} \ddot{F}^{k l, r s}\right) \nabla_{i} h_{k l} \nabla_{j} h_{r s}+\left\langle X, e_{k}\right\rangle \nabla^{k} G .
$$

In particular, setting $G=\frac{Q}{F}$ where $Q(\mathcal{W})=q(\kappa)$ is symmetric and homogeneous of degree 1 , the equation for $G$ may be rewritten as

$$
\mathcal{L} G=\frac{1}{F}\left(\dot{F}^{i j} \ddot{Q}^{k l, r s}-\dot{Q}^{i j} \ddot{F}^{k l, r s}\right) \nabla_{i} h_{k l} \nabla_{j} h_{r s}-\frac{2}{F} \dot{F}^{i j} \nabla_{i} G \nabla_{j} F+\left\langle X, e_{k}\right\rangle \nabla^{k} G .
$$

Now the $\ddot{F}$ term will have a sign provided $\dot{Q}$ has a sign; we require this to be the same sign as the $\ddot{Q}$ term. For the case of $F$ concave, set $Q=|A|+\eta H$, where

$$
\eta>-\min _{M} \frac{\kappa_{\min }}{|A|} .
$$

(In fact $\eta=0$ would suffice in the case of $M$ convex.) We have

$$
\frac{\partial q}{\partial \kappa_{i}}=\frac{\kappa_{i}}{|A|}+\eta,
$$

so the condition on $\eta$ ensures $\dot{Q}$ is everywhere positive definite on $M$. Furthermore, $Q$ is strictly convex in non radial directions, so since $\dot{F}$ is also positive definite and attains a minimum on $M$, we have adopting suitable coordinates

$$
\dot{F}^{i j} \ddot{Q}^{k l, r s} \nabla^{i} h_{p q} \nabla_{i} h_{k l} \geq \min _{j}\left(\min _{M} \frac{\partial f}{\partial \kappa_{j}}\right) \sum_{i} \ddot{Q}^{k l, r s} \nabla_{i} h_{p q} \nabla_{i} h_{k l} \geq C \frac{|\nabla A|^{2}}{|A|}
$$

using again the result [4, Lemma 7.12].

By the same reasoning as above observe that it is not possible for $\frac{Q}{F}$ to be identically constant on $M$ unless $M$ is a sphere.

In the case of $F$ convex, a similar argument using $Q=\eta H-|A|$, where $\eta>\max _{M} \frac{\kappa_{\max }}{|A|}$ gives the result.

\section{Proof of Theorem 1.5}

Since $M$ is smooth and strictly convex, in this case we may parametrise $M$ using the Gauss map. Denote by $\Omega$ the convex region of $\mathbb{R}^{n+1}$ bounded by $M$. Then $M$ has support function $s: \mathbb{S}^{n} \rightarrow \mathbb{R}$ defined by

$$
s(z)=\sup \{\langle x, z\rangle: x \in \Omega\},
$$


and $M$ is the image of the embedding

$$
X(z)=s(z) z+\bar{\nabla} s(z) .
$$

This map takes $z$ to the unique point of $M$ with normal direction $z$. Throughout this section we denote by $\bar{\nabla}$ the standard connection on $\mathbb{S}^{n}$ and by $\bar{g}_{i j}$ the standard metric on $\mathbb{S}^{n}$. The matrix of the inverse Weingarten map of $M, \mathcal{W}^{-1}=\left(r_{i j}\right)$ can be written in terms of the support function and its second derivatives:

$$
r_{i j}=\bar{\nabla}_{i} \bar{\nabla}_{j} s+\bar{g}_{i j} s
$$

Writing $\Phi\left(\mathcal{W}^{-1}\right)=F(\mathcal{W})^{-1}$, with $\alpha=1$ equation (1.3) becomes

$$
s=\Phi\left(\mathcal{W}^{-1}\right)^{-1}
$$

Following a similar procedure as in the previous proof, but differentiating on the sphere, we compute that

$$
\overline{\mathcal{L}} r_{i j}=-\ddot{\Phi}^{k l, r s} \bar{\nabla}_{i} r_{k l} \bar{\nabla}_{j} r_{r s}+2 \Phi^{-1} \bar{\nabla}_{i} \Phi \bar{\nabla}_{j} \Phi+\left(\dot{\Phi}^{k l} \bar{g}_{k l}-\Phi^{2}\right) r_{i j}
$$

and

$$
\overline{\mathcal{L}} \Phi=2 \Phi^{-1} \dot{\Phi}^{k l} \bar{\nabla}_{k} \Phi \bar{\nabla}_{l} \Phi+\left(\dot{\Phi}^{k l} \bar{g}_{k l}-\Phi^{2}\right) \Phi,
$$

where $\overline{\mathcal{L}}=\dot{\Phi}^{k l} \bar{\nabla}_{k} \bar{\nabla}_{l}$. Now, setting $T_{i j}=r_{i j}-C \Phi \bar{g}_{i j}$ we have for any constant $C$,

$$
\begin{aligned}
\overline{\mathcal{L}} T_{i j}= & -\ddot{\Phi}^{k l, r s} \bar{\nabla}_{i} r_{k l} \bar{\nabla}_{j} r_{r s}+2 \Phi^{-1} \bar{\nabla}_{i} \Phi \bar{\nabla}_{j} \Phi \\
& -2 C \Phi^{-1} \dot{\Phi}^{k l} \bar{\nabla}_{k} \Phi \bar{\nabla}_{l} \Phi \bar{g}_{i j}+\left(\dot{\Phi}^{k l} \bar{g}_{k l}-\Phi^{2}\right) T_{i j} .
\end{aligned}
$$

Considering $T$ as a function on $\left\{(p, v) \in \mathbb{R}^{n+1} \times \mathbb{R}^{n+1}:|p|=1,|v|=1, p \perp v\right\}$, that is, for $p \in \mathbb{S}^{n}$ and $v \in T_{p} \mathbb{S}^{n}$ with length 1 ,

$$
T(p, v)=T_{i j} v^{i} v^{j},
$$

$T$ attains a minimum and a maximum by compactness. Choose $C$ such that $T_{i j} \leq 0$ everywhere and $T_{i j} v^{i} v^{j}=0$ at some $p$. We obtain a contradiction to the maximum principle applied to (3.2) by estimating the derivative terms as in [8]. As in that paper, or in [11], it suffices to show that for any symmetric 3-tensor $B_{i j k}$ with $\left(B_{k i j}-C \delta_{i j} \dot{\Phi}^{p q} B_{k p q}\right) v^{i} v^{j}=0$, we have the inequality

$$
\begin{gathered}
\left(\ddot{\Phi}^{k l, p q}-2 \Phi^{-1} \dot{\Phi}^{k l} \dot{\Phi}^{p q}\right) B_{i k l} B_{j p q} v^{i} v^{j}+2 C \Phi^{-1} \dot{\Phi}^{i j} \dot{\Phi}^{k l} \dot{\Phi}^{p q} B_{i k l} B_{j p q}|v|^{2} \\
+2 \dot{\Phi}^{k l}\left[2 \Gamma_{k}^{p}\left(B_{l i p}-C \delta_{i p} \dot{P} h i^{r s} B_{l r s}\right) v^{i}-\Gamma_{k}^{p} \Gamma_{l}^{q} T_{p q}\right] \leq 0
\end{gathered}
$$


for some choice of tensor $\Gamma$. It also suffices to assume all eigenvalues of $r_{i j}$ are distinct. Choosing coordinates at $p$ such that $r_{i j}$ is diagonal and $v=e_{1}$, we have at $p, r_{1}=r_{11}=C \Phi$ and $r_{1 j}=0, j>1$. The condition for $B$ becomes: for all $k$, $B_{k 11}=C \dot{\Phi}^{p q} B_{k p q}, B_{k 1 j}=0, j \geq 2$, and the terms in (3.3) can be rewritten as

$$
\begin{aligned}
\ddot{\Phi}^{k l, p q} B_{1 k l} B_{1 p q}= & \sum_{k, p} \ddot{\varphi}^{k, p} B_{1 k k} B_{1 p p}+2 \sum_{k>1} \frac{\dot{\varphi}^{1}-\dot{\varphi}^{k}}{r_{1}-r_{k}} B_{11 k}^{2} \\
& +2 \sum_{1<k<l} \frac{\dot{\varphi}^{k}-\dot{\varphi}^{l}}{r_{k}-r_{l}} B_{1 k l}^{2}, \\
-2 \Phi^{-1} \dot{\Phi}^{k l} \dot{\Phi}^{p q} B_{1 k l} B_{1 p q}= & -\frac{2}{C^{2} \Phi} B_{111}^{2} \\
2 C \Phi^{-1} \dot{\Phi}^{i j} \dot{\Phi}^{k l} \dot{\Phi}^{p q} B_{i k l} B_{j p q}= & \frac{2}{C \Phi} \dot{\varphi}^{k} B_{k 11}^{2}
\end{aligned}
$$

and, choosing $\Gamma_{k}^{1}=0$ for all $k$,

$$
\begin{aligned}
2 \dot{\Phi}^{k l} & {\left[2 \Gamma_{k}^{p}\left(B_{l i p}-C \delta_{i p} \dot{P} h i^{r s} B_{l r s}\right) v^{i}-\Gamma_{k}^{p} \Gamma_{l}^{q} T_{p q}\right] } \\
& =-2 \sum_{k=1}^{n} \sum_{p=2}^{n} \frac{\dot{\varphi}^{k}}{r_{1}-r_{p}} B_{1 k p}^{2}+2 \sum_{k=1}^{n} \sum_{p=2}^{n} \dot{\varphi}^{k}\left(r_{1}-r_{p}\right)\left(\Gamma_{k}^{p}+\frac{B_{1 k p}^{2}}{r_{1}-r_{p}}\right)^{2} .
\end{aligned}
$$

We remark that the formula for writing $\ddot{\Phi}$ in terms of first and second derivatives of $\varphi$ may be found, for example, in [8].

We use the above to show that the coefficient of each $B_{i j k}^{2}$ term is nonpositive. It is obviously best to choose $\Gamma_{k}^{p}=-\frac{B_{1 k p}^{2}}{r_{1}-r_{p}}$. Now, the $\ddot{\varphi}$ term is clearly nonpositive by concavity, so the terms here are taken care of. The coefficient of all the remaining $B_{111}^{2}$ terms is

$$
-\frac{2}{C^{2} \Phi}+\frac{2}{C \Phi} \dot{\varphi}^{1}=\frac{2}{C^{2} \Phi^{2}}\left(-\varphi+\dot{\varphi}^{1} r_{1}\right) \leq 0
$$

in view of Conditions 1.2 and the Euler identity. Above we also used that $r_{1}=C \varphi$ at $p$. Next, the coefficient of all the remaining $B_{11 k}^{2}$, for each $k \geq 2$, is

$$
2 \frac{\dot{\varphi}^{1}-\dot{\varphi}^{k}}{r_{1}-r_{k}}+\frac{2}{C \Phi} \dot{\varphi}^{k}-\frac{2 \dot{\varphi}^{k}}{r_{1}-r_{k}}=\frac{-2 \dot{\varphi}^{k} r_{k}}{r_{1}\left(r_{1}-r_{k}\right)} \leq 0
$$

since $\dot{\varphi}^{k}>0$ for each $k$ and $M$ is convex. The coefficient of the remaining $B_{1 k k}^{2}$ for $k>1$ is

$$
\frac{-2 \dot{\varphi}^{k}}{r_{1}-r_{k}}<0
$$


while the coefficient of $B_{1 k l}^{2}$, for $1<k<l$, is

$$
2 \frac{\dot{\varphi}^{k}-\dot{\varphi}^{l}}{r_{k}-r_{l}}-\frac{2 \dot{\varphi}^{k}}{r_{1}-r_{l}}-\frac{2 \dot{\varphi}^{l}}{r_{1}-r_{k}} .
$$

The first of these terms is nonpositive by concavity of $\varphi$, while the other two are clearly negative.

In view of (3.2), $T_{i j} v^{i} v^{j}$ cannot attain a maximum unless there is a parallel vector field $v=e_{1}$ such that $T_{i j} v^{i} v^{j} \equiv 0$. This means that each nonpositive term above must be identically equal to zero. In particular,

$$
0 \equiv B_{k 11}=\bar{\nabla}_{k} r_{11}=C \bar{\nabla}_{k} \Phi
$$

so $\Phi$ is identically constant on $M$. It follows by Aleksandrov's theorem [2] that $M$ is a sphere.

\section{Proof of Theorem 1.6}

For convex surfaces we may remove the second derivative requirement on $F$ by an argument as in [9]. We need only assume $F$ satisfies Conditions 1.2 and $\alpha \geq 1$. If $\alpha>1$ we do need $M$ to satisfy the pinching condition of the theorem as well. For consistency with [9] it is useful to define here $\underline{F}=F^{\alpha}$ and compute directly with this homogeneous of degree $\alpha$ quantity.

As in [9], we consider the equation for the quantity

$$
G(\mathcal{W})=\frac{2|A|^{2}-H^{2}}{H^{2}}
$$

also written in terms of the principal curvatures as

$$
g\left(\kappa_{1}, \kappa_{2}\right)=\frac{\left(\kappa_{1}-\kappa_{2}\right)^{2}}{\left(\kappa_{1}+\kappa_{2}\right)^{2}}
$$

$G$ is symmetric, homogeneous of degree zero, nonnegative and identically equal to zero if and only if $M$ is a sphere. We compute that

$$
\frac{\partial g}{\partial \kappa_{1}}=\frac{4\left(\kappa_{1}-\kappa_{2}\right) \kappa_{2}}{H^{3}} \quad \text { and } \quad \frac{\partial g}{\partial \kappa_{2}}=\frac{4\left(\kappa_{2}-\kappa_{1}\right) \kappa_{1}}{H^{3}}
$$

Since

$$
\nabla_{i} \nabla_{j} G=\ddot{G}^{k l, p q} \nabla_{i} h_{k l} \nabla_{j} h_{p q}+\dot{G}^{k l} \nabla_{i} \nabla_{j} h_{p q},
$$

we have by contraction with $\underline{\dot{F}}^{i j}$,

$$
\mathcal{L} G=\underline{\dot{F}}^{i j} \ddot{G}^{k l, p q} \nabla_{i} h_{k l} \nabla_{j} h_{p q}+\underline{\dot{F}}^{i j} \dot{G}^{k l} \nabla_{i} \nabla_{j} h_{p q} .
$$


Contracting (2.1), with $\dot{G}^{i j}$, with $\underline{F}$ replacing $F$, we also have

$$
\dot{G}^{i j} \underline{\underline{F}}^{k l, p q} \nabla_{i} h_{k l} \nabla_{j} h_{p q}+\dot{G}^{i j} \underline{\dot{F}}^{k l} \nabla_{i} \nabla_{j} h_{k l}=-\underline{F} \dot{G}^{i j} h_{i}{ }^{k} h_{k j}+\left\langle X, e_{k}\right\rangle \nabla^{k} G .
$$

By interchanging covariant derivatives, in view of the homogeneity of $\underline{F}$ and of $G$,

$$
\underline{\dot{F}}^{i j} \dot{G}^{k l} \nabla_{i} \nabla_{j} h_{k l}=\underline{\dot{F}}^{i j} \dot{G}^{k l} \nabla_{k} \nabla_{l} h_{i j}+\alpha \underline{F} \dot{G}^{k l} h_{k m} h_{l}^{m}
$$

so using (4.1) and (4.2) we find that $G$ satisfies

$$
\begin{aligned}
\mathcal{L} G= & \left(\dot{\dot{F}}^{i j} \ddot{G}^{k l, p q}-\dot{G}^{i j} \ddot{F}^{k l, p q}\right) \nabla_{i} h_{k l} \nabla_{j} h_{p q} \\
& +(\alpha-1) \underline{F} \dot{G}^{k l} h_{k m} h_{l}^{m}+\left\langle X, e_{k}\right\rangle \nabla^{k} G .
\end{aligned}
$$

Suppose $G$ attains a maximum at some point $p$ of $M$. If this maximum is $G=0$ then $G$ is identically zero and $M$ is a sphere. So suppose $G>0$ at $p$. Choose coordinates at $p$ such that $g_{i j}=\delta_{i j}$ and $\left(h_{i j}\right)$ is a diagonal matrix. By the argument in [9], in view of the homogeneity of $\underline{F}$ and $G$ and convexity of $M$, the entire $\nabla A$ term is nonnegative (this requires the additional pinching assumption if $\alpha>1$ ). We also have at $p$

$$
\dot{G}^{k l} h_{k m} h_{l}^{m}=\sum_{i} \frac{\partial g}{\partial \kappa_{i}} \kappa_{i}^{2}=\frac{4 \kappa_{1} \kappa_{2}\left(\kappa_{1}-\kappa_{2}\right)^{2}}{H^{3}}>0,
$$

by convexity of $M$. But then $G$ having a maximum at $p$ contradicts (4.3). Therefore $G$ is identically constant. If $\alpha>1$ the zero order term of (4.3) gives immediately that $M$ is a sphere. If $\alpha=1$ then this term does not appear, but the gradient term, as in [9], is equal to

$$
\frac{8 f}{H^{3}}\left\{\left(\nabla_{1} h_{22}\right)^{2}+\left(\nabla_{2} h_{11}\right)^{2}\right\} \equiv 0 .
$$

Hence $\nabla_{1} h_{22} \equiv 0$ and $\nabla_{2} h_{11} \equiv 0$. That $\nabla A \equiv 0$ now follows from the fact that $\nabla G \equiv 0$. Therefore again $M$ is a sphere.

\section{Proof of Theorem 1.7}

Here we will need the following estimates:

Lemma 5.1. Let $M$ be a closed, convex, $n$-dimensional hypersurface with pinched principal curvatures in the sense of (1.5). Then the curvature of $M$ also satisfies:

(i) $\left|A^{0}\right|^{2} \leq\left(\frac{n-1}{2}\right)(1-\varepsilon)^{2} H^{2}$,

(ii) $H C-\left(|A|^{2}\right)^{2} \geq \frac{\varepsilon^{2}}{n} H^{2}\left|A^{0}\right|^{2}$,

(iii) $\left|H \nabla_{i} h_{j k}-h_{j k} \nabla_{i} H\right|^{2} \geq\left(\frac{n-1}{2 n^{2}}\right) \varepsilon^{2} H^{2}|\nabla A|^{2}$. 
The first of the above results follows by straightforward calculation. The second was proved in [20], while the third, attributed to Huisken, appears in [13]. Our constants in (ii) and (iii) above are different from those in the earlier papers because of our different definition of $\varepsilon$.

Proof of Theorem 1.7. This proof is again along the lines of Huisken [21]. However, complications arise since the speed is not $H$; we need hypersurface $M$ sufficiently curvature pinched, Conditions 1.2 on $F$ and we take $\alpha \geq 1$.

From (1.3) we compute for a general symmetric function of the curvature $G(\mathcal{W})=g(\kappa(\mathcal{W}))$

$$
\begin{aligned}
\alpha F^{\alpha-1} \mathcal{L} G= & -\left(\ddot{F^{\alpha}}\right)^{k l, r s} \dot{G}^{i j} \nabla_{i} h_{k l} \nabla_{j} h_{r s}+\left(\dot{F}^{\alpha}\right)^{i j} \ddot{G}^{k l, r s} \nabla_{i} h_{k l} \nabla_{j} h_{r s} \\
& +\left\langle X, e_{k}\right\rangle \nabla^{k} G+\left[1-\left(\dot{F}^{\alpha}\right)^{k l} h_{k m} h_{l}^{m}\right] \dot{G}^{i j} h_{i j} \\
& +(\alpha-1) F^{\alpha} \dot{G}^{i j} h_{i m} h_{j}^{m}
\end{aligned}
$$

and with $G=\frac{H^{2}}{|A|^{2}}$, which is homogeneous of degree 0 ,

$$
\begin{aligned}
\frac{\alpha}{2}|A|^{4} F^{\alpha-1} \mathcal{L}\left(\frac{H^{2}}{|A|^{2}}\right)= & -H\left(\ddot{F}^{\alpha}\right)^{k l, r s}\left(|A|^{2} g^{i j}-H h^{i j}\right) \nabla_{i} h_{k l} \nabla_{j} h_{r s} \\
& +\frac{|A|^{4}}{H}\left(\dot{F^{\alpha}}\right)^{i j} \nabla_{i} H \nabla_{j}\left(\frac{H^{2}}{|A|^{2}}\right) \\
& -\left(\dot{F^{\alpha}}\right)^{i j}\left(H \nabla_{i} h_{k l}-h_{k l} \nabla_{i} H\right)\left(H \nabla_{j} h^{k l}-h^{k l} \nabla_{j} H\right) \\
& +\frac{1}{2}|A|^{4}\left\langle X, e_{k}\right\rangle \nabla^{k}\left(\frac{H^{2}}{|A|^{2}}\right) \\
& -(\alpha-1) F^{\alpha} H\left[H C-\left(|A|^{2}\right)^{2}\right] .
\end{aligned}
$$

Observe that since $\alpha \geq 1$, the zero order term in (5.1) is nonpositive from Lemma 5.1, (ii). Since $F$ is homogeneous of degree 1 , for the $\ddot{F}^{\alpha}$ term we have

$$
\begin{aligned}
& -H\left(\ddot{F}^{\alpha}\right)^{k l, r s}(\mathcal{W})\left(|A|^{2} g^{i j}-H h^{i j}\right) \nabla_{i} h_{k l} \nabla_{j} h_{r s} \\
& =-H|A|^{\alpha-2}\left(\ddot{F}^{\alpha}\right)^{k l, r s}\left(\frac{\mathcal{W}}{|A|}\right)\left(|A|^{2} g^{i j}-H h^{i j}\right) \nabla_{i} h_{k l} \nabla_{j} h_{r s} \\
& \leq \sqrt{n} H|A|^{\alpha-1}\left|A^{0}\right| M_{2}(\varepsilon)|\nabla A|^{2},
\end{aligned}
$$


while for the norm-like $\dot{F}^{\alpha}$ term,

$$
\begin{aligned}
& \left(\dot{F}^{\alpha}\right)^{i j}(\mathcal{W})\left(H \nabla_{i} h_{k l}-h_{k l} \nabla_{i} H\right)\left(H \nabla_{j} h^{k l}-h^{k l} \nabla_{j} H\right) \\
& =|A|^{\alpha-1}\left(\dot{F}^{\alpha}\right)^{i j}\left(\frac{\mathcal{W}}{|A|}\right)\left(H \nabla_{i} h_{k l}-h_{k l} \nabla_{i} H\right)\left(H \nabla_{j} h^{k l}-h^{k l} \nabla_{j} H\right) \\
& \geq M_{1}(\varepsilon)|A|^{\alpha-1}|H \nabla A-A \nabla H|^{2} \\
& \geq\left(\frac{n-1}{2 n^{2}}\right) \varepsilon^{2} H^{2}|A|^{\alpha-1} M_{1}(\varepsilon)|\nabla A|^{2} .
\end{aligned}
$$

Here we have used the following:

$$
M_{2}(\varepsilon)=\sup \left\{\left|D^{2} f^{\alpha}(\kappa)(\xi, \xi)\right|: \kappa \in K_{\varepsilon},|\kappa|=1,|\xi|=1\right\}
$$

and

$$
M_{1}(\varepsilon)=\inf \left\{\frac{\partial f^{\alpha}}{\partial \kappa_{i}}(\kappa): 1 \leq i \leq n, \kappa \in K_{\varepsilon},|\kappa|=1\right\},
$$

where for any $\varepsilon \in[0,1]$,

$$
K_{\varepsilon}=\left\{\kappa \in \Gamma^{+}: \kappa_{i} \geq \varepsilon \kappa_{j} \text { for all } 1 \leq i, j \leq n\right\} .
$$

Observe that $M_{2}$ and $M_{1}$ are positive and finite as taken over a compact set. In particular, $M_{1}(\varepsilon)$ is attained as a positive minimum and is nondecreasing in $\varepsilon$. Also $M_{2}$ is clearly positive and nonincreasing in $\varepsilon$.

Substituting the above estimates into (5.1) and using Lemma 5.1 we find

$$
\frac{\alpha}{2}|A|^{4} F^{\alpha-1} \mathcal{L}\left(\frac{H^{2}}{|A|^{2}}\right) \leq Q(\varepsilon) H^{2}|A|^{\alpha-1}|\nabla A|^{2}+\frac{1}{2}|A|^{4}\left\langle X, e_{k}\right\rangle \nabla^{k}\left(\frac{H^{2}}{|A|^{2}}\right),
$$

where

$$
Q(\varepsilon)=\sqrt{\frac{n-1}{2}}(1-\varepsilon) M_{2}(\varepsilon)-\left(\frac{n-1}{2 n^{2}}\right) \varepsilon^{2} M_{1}(\varepsilon) .
$$

A direct computation shows that $Q(\varepsilon)$ is monotone nonincreasing in $\varepsilon$. Furthermore, $Q(\varepsilon)>0$ for small $\varepsilon$ (weaker pinching) and $Q(\varepsilon)<0$ for large $\varepsilon$ close to 1 (strong pinching). It follows that there is a weakest pinching ratio $\varepsilon$ such that, $M$ satisfying such pinching gives rise to

$$
\frac{\alpha}{2}|A|^{4} F^{\alpha-1} \mathcal{L}\left(\frac{H^{2}}{|A|^{2}}\right) \leq 0
$$

The strict elliptic maximum principle then gives, under this pinching, $|\nabla A| \equiv 0$ so $M$ is a sphere. 


\section{References}

[1] U. ABRESCH and J. LANGER, The normalized curve shortening flow and homothetic solutions, J. Differential Geom. 23 (1986), 175-196.

[2] A. D. Aleksandrov, Uniqueness theorems for surfaces in the large. V, Vestnik Leningrad. Univ. 13 (1958), 5-8.

[3] K. ANADA, Contraction of surfaces by harmonic mean curvature flows and nonuniqueness of their self similar solutions, Calc. Var. Partial Differential Equations 12 (2001), 109-116.

[4] B. H. ANDREws, Contraction of convex hypersurfaces in Euclidean space, Calc. Var. Partial Differential Equations 2 (1994), 151-171.

[5] B. H. ANDREws, Contraction of convex hypersurfaces by their affine normal, J. Differential Geom. 43 (1996), 207-230.

[6] B. H. ANDREWS, Gauss curvature flow: the fate of the rolling stones, Invent. Math. 138 (1999), 151-161.

[7] B. H. ANDREWs, Motion of hypersurfaces by Gauss curvature, Pacific J. Math. 195 (2000), $1-34$.

[8] B. H. ANDREws, Pinching estimates and motion of hypersurfaces by curvature functions, J. Reine Angew. Math. 608 (2007), 17-33.

[9] B. H. ANDREWS, Moving surfaces by non-concave curvature functions, Calc. Var. Partial Differential Equations 39 (2010), 649-657.

[10] B. H. ANDREWS and J. A. MCCOY, Convex hypersurfaces with pinched principal curvatures and flow of convex hypersurfaces by high powers of curvature, Trans. Amer. Math. Soc., to appear.

[11] B. H. ANDREws, J. A. MCCOY and Z. YU, Contraction of nonsmooth convex hypersurfaces into spheres, available at arxiv.org/abs/1104.0756.

[12] S. Angenent, Shrinking doughnuts, Progr. Nonlinear Differential Equations Appl. 7 (1992), Birkhäuser, 21-38.

[13] E. Cabezas-Rivas and C. Sinestrari, Volume-preserving flow by powers of the $\mathrm{m}$ th mean curvature, Calc. Var. Partial Differential Equations 38 (2010), 441-469.

[14] E. CAlabi, Complete affine hyperspheres, I, Sympos. Math. 10 (1971/72), 19-38 (1972).

[15] B. CHOw, Deforming convex hypersurfaces by the $\mathrm{n}$ th root of the Gaussian curvature, J. Differential Geom. 22 (1985), 117-138.

[16] B. CHOw, Deforming convex hypersurfaces by the square root of the scalar curvature, Invent. Math. 87 (1987), 63-82.

[17] C. GERHARDT, Flow of nonconvex hypersurfaces into spheres, J. Differential Geom. 32 (1990), 299-314.

[18] C. Gerhardt, "Curvature Problems", Series in Geometry and Topology, Vol. 39, International Press, Somerville, 2006.

[19] Q. HAN, Deforming convex hypersurfaces by curvature functions, Analysis 17 (1997), 113127.

[20] G. Huisken, Flow by mean curvature of convex surfaces into spheres, J. Differential Geom. 20 (1984), 237-266.

[21] G. HuISKEN, Asymptotic behavior for singularities of the mean curvature flow, J. Differential Geom. 31 (1990), 285-299.

[22] G. M. Lieberman, "Second Order Parabolic Differential Equations", World Scientific, Singapore, 1996.

[23] O. C. SCHNÜRER, Surfaces contracting with speed $|A|^{2}$, J. Differential Geom. 71 (2005), 347-363.

[24] F. SChulze, Convexity estimates for flows by powers of the mean curvature, Ann. Sc. Norm. Super. Pisa Cl. Sci. (5) 5 (2006), 261-277.

[25] K. SMOCZYK, Harnack inequalities for curvature flows depending on mean curvature, New York J. Math. 3 (1997), 103-118 (electronic). 
[26] K. Sмосzүк, Self-shrinkers of the mean curvature flow in arbitrary codimension, Int. Math. Res. Not. 48 (2005), 2983-3004.

[27] K.-S. Tso, Deforming a hypersurface by its Gauss-Kronecker curvature, Comm. Pure Appl. Math. 38 (1985), 867-882.

[28] J. I. E. URBAS, An expansion of convex hypersurfaces, J. Differential Geom. 33 (1991), 91-125.

[29] J. I. E. URBAS, On the expansion of starshaped hypersurfaces by symmetric functions of their principal curvatures, Math. Z. 205 (1990), 355-372.

Institute for Mathematics and its Applications School of Mathematics and Applied Statistics University of Wollongong Wollongong, NSW 2522, Australia jamesm@uow.edu.au 